\title{
Fault Diagnosis in Multi Phase Induction Machine using Mind Evolution Computation Algorithm Optimized Neural Network
}

\author{
Balamurugan Annamalai, Sivakumaran Thangavel Swaminathan
}

\begin{abstract}
This article proposes a new solution method for diagnosing faults in a multi phase induction motor using least mean square filter (LMS) and a new hybrid neural network with mind evolution computation algorithm. The entire procedure for teaching an artificial neural network (ANN) is popularly thought of among the toughest activities in system learning and also it has lately attracted lots of research workers. The proposed hybrid fault diagnosing method includes an efficient feature extractor based on LMS and a fault classifier based on a hybrid neural network. First, the LMS method is used to extract the effective features. The mind evolution computation algorithm is employed to train the neural network. The performance and efficiency of the presented hybrid neural network classifier is estimated by testing a total of 600 samples, which are modeled on the basis of the failure model. The average correct classification with and without mind evolution computation algorithm is about $98 \%$ and $96.17 \%$ for various fault signals respectively. The outcome got from the simulation analysis shows the potency of the proposed hybrid neural network for fault diagnosis in multi phase induction motor.
\end{abstract}

Keywords : Fault diagnosis, feature extraction, least mean square, multi layer perceptron neural network, mind evolution computation algorithm.

\section{INTRODUCTION}

$\mathrm{T}$

he multiphase induction motor's fault investigation and identification are essential in products diagnosis procedure. On account of construction faculties of motors and its working theory, fault diagnosis processes and the identification procedures have many characteristics [1].

A fault in electrical engine commonly has lots of outward symptoms, as an instance, as soon as a bar of motor has been broken, and lots of symptoms correlated each other occur, for example, instance shaking increment, start up time prolongation, current swing of stator, slip augmentation, speed rate and torque undulation, temperature shift, etc [2]. Once a bar is broken and the motor continues to run, the

Revised Manuscript Received on February 05, 2020.

* Correspondence Author

Balamurugan Annamalai*, Research Scholar, Dept. of EEE, Sathyabama Institute of Science and Technology, Tamil Nadu, India. Email: at.balamurugan@gmail.com

Sivakumaran Thangavel Swaminathan, Professor \& Principal, Dept. of EEE, Sasurie College of Engineering, Tiruppur, Tamil Nadu, India.

(C) The Authors. Published by Blue Eyes Intelligence Engineering and Sciences Publication (BEIESP). This is an open access article under the CC BY-NC-ND license (http://creativecommons.org/licenses/by-nc-nd/4.0/)

range of broken bar will grow up, outward symptoms are far more and more noticeable, and malfunction eventually become much more and more acute; and also finally motor is going to probably likely soon undoubtedly be destroyed. On the flip side, same symptoms are caused by quite many faults.

Many circumstance changes may cause electric-motor malfunction, for example, loading variation and other motor functionality that all types of signs and symptoms; hence the relation is very difficult [3]. For this reason, it's quite tricky to identify motor's fault. For very long period, processes and several identification methods are obtained for example current analysis, vibration evaluation, thermal analysis and therefore forth. Some skilled strategies for fault identification of multiphase induction motor are grown [4]. At the moment, exploration fault investigation and identification process continues to be a substantial problem due to the fact that (i) the relationship between fault reason and symptom is quite sophisticated; (ii) the convenience of fault identification procedure for multiphase induction motor is quite confined; (iii) the artificial-intelligence identification procedure predicated on principle discursion, you can find lots of issues like comprehension expression and receiving, principle suit, etc. The procedure and the basic theory of fault identification of multiphase induction motor are all discussed $[5,6]$. To the grounds of this study, the motor voltage and its slope are used as the features to diagnosis the fault in multiphase induction motor; and a identification system based on neural network has been now exhibited. By motor's state parameters, the procedure could recognize the different fault. This proposed approach is smart, dependable and accurate. The application of an artificial neural network (ANN) has stood out as a facilitating mechanism in solving problems in many areas [7]. In this perspective, a study was carried out through the implementation and analysis of radial basis function (RBF) neural network and multilayer perceptron (MLP), with the objective of comparing the results based on quantitative procedures, with emphasis on training and testing, assisting in the classification of fault in induction motor [8]. The article demonstrates the problems of diagnosing asynchronous motors in case of a malfunction of the rotor, stator and shaft bearing $[9,10]$. For diagnostic purposes, methods of artificial intelligence on the basis of ANN are used. A feed forward neural network (FFNN) is used [8]. In this work, the ANN is trained and tested with the motor voltage and its slope. The effectiveness of the developed FFNN is estimated for fault diagnosis in multiphase induction motor.

Published By: 
The article is organized as follows. In section 2, a preliminary least mean square (LMS) algorithm is given and then the execution of the presented feature extraction using LMS is depicted. A brief introduction about FFNN is given in the third section. The section 4 gives the implementation of the mind evolution computational algorithm (MECA) in training the MLP. The section 5 depicts the results of modeling and analysis and shows the outcomes of classification technique. Finally, the findings are given in section 6 .

\section{FEATURE EXTRACTION}

\section{A. LMS Algorithm}

The LMS procedure for signal attribute extraction is represented in Fig. 1, at which $\mathrm{y}_{\mathrm{t}}$ refers the true signal, $\hat{\mathrm{y}}_{t}$ describes the signal esteem and $\mathrm{X}_{\mathrm{t}}=\left[\mathrm{x}_{0 \mathrm{t}}, \mathrm{x}_{1 \mathrm{t}}, \ldots, \mathrm{x}_{\mathrm{N}-1 \mathrm{t}}\right]^{\mathrm{T}}$ is the input signal vector in the $t^{\text {th }}$ moment. The filter can estimate accurately the signal having the right significance of its own $\mathrm{W}_{\mathrm{t}}$, and it is accessed through diminishing the squared of this error signal $e_{t}[11]$. So awareness is gained by the frame that will be symbolized like a trained filter in which the filter coefficients are accommodated in a fashion in their esteems. At each iteration, the weight vector $\mathrm{W}_{\mathrm{t}}$ is figured as,

$W_{k+1}=W_{k}+\mu\left(-\nabla_{\mathrm{k}}\right)$

At which $\mathrm{W}_{\mathrm{t}}=\left[\mathrm{w}_{0 \mathrm{t}}, \mathrm{w}_{1 \mathrm{t}}, \ldots, \mathrm{w}_{\mathrm{N}-1 \mathrm{t}}\right]^{\mathrm{T}}$ is the filter coefficient, $\mu$ is the adaptation parameter and $\nabla_{t}$ can be actually the gradient of this error execution surface with regard to filter coefficient, this is figured as,

$\widehat{\nabla}_{k}=-2 e_{k} X_{k}$

Even the recursion (1) is popularly known as the LMS approach and also it is initialized by setting to zero. The procedure is started by calculating the error signal $e_{t}$, and it is required to compute the coefficient. Until the stability states have been accomplished, the following procedure is implemented. The stableness of the this loop system is handled with the parameter $\mu$ and also it needs to match the following standards,

$0<\mu<\frac{2}{\text { Total input power }}$

At which $\mu$ the input power cites to the total amount of the mean square value of these input signals. The LMS strategy absorbs moment to learn regarding its own input with minimum mean square error when $\mu$ is little and vice versa. Hence, a time measure step sized ordering of $\mu$ is needed for convergence [11].

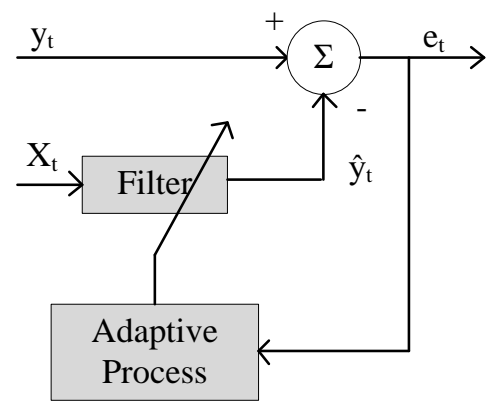

Fig. 1. LMS Filter

\section{B. Feature Extraction using LMS}

The voltage signal of an induction motor could be shown in discrete manner as,

$$
\begin{aligned}
& V_{a_{k}}=V_{m} \cos (\omega k \Delta T+\varphi)+\epsilon_{a_{k}} \\
& V_{b_{k}}=V_{m} \cos \left(\omega k \Delta T+\varphi-\frac{2 \pi}{3}\right)+\epsilon_{b_{k}} \\
& V_{c_{k}}=V_{m} \cos \left(\omega k \Delta T+\varphi+\frac{2 \pi}{3}\right)+\epsilon_{c_{k}}
\end{aligned}
$$

where, $\mathrm{V}_{\mathrm{m}}$ is the fundamental component's maximum magnitude, $\epsilon_{t}$ is the noise found in the voltage waveform, $t$ is the sampling period, $\varphi$ is the fundamental component's phase, and $\omega$ is the voltage's angular frequency. The complex form of signal arrived from the motor voltage is got by $\alpha \beta$ transformation [11] as stated as follows,

$\left[\begin{array}{l}V_{\alpha_{k}} \\ V_{\beta_{k}}\end{array}\right]=\sqrt{\frac{2}{3}}\left[\begin{array}{ccc}1 & -\frac{1}{2} & -\frac{1}{2} \\ 0 & \frac{\sqrt{3}}{2} & -\frac{\sqrt{3}}{2}\end{array}\right]\left[\begin{array}{lll}V_{a_{k}} & V_{b_{k}} & V_{c_{k}}\end{array}\right]^{T}$

The complex voltage can be gotten as,

$V_{k}=V_{\alpha_{k}}+j V_{\beta_{k}}$

The voltage $\mathrm{V}_{\mathrm{t}}$ can be modelled as,

$V_{k}=A e^{j(\omega k \Delta T+\varphi)}+\xi_{k}$

$V_{k}=\widehat{V}_{k}+\xi_{k}$

In which, $\mathrm{A}$ is the amplitude of this signal $\mathrm{V}_{\mathrm{t}}$, and $\xi_{t}$ is voltage's noise component and $\widehat{V}_{t}=A e^{j(\omega t \Delta T+\varphi)}$.

The voltage could be modelled as,

$\widehat{V_{k}}=\widehat{V}_{k}-1^{e^{j \omega \Delta T}}$

This version is also used in the proposed attribute estimation and the scheme that describes the extraction method is depicted in Fig. 2. The error signal $e_{t}$ in this particular circumstance is figured as,

$e_{k}=V_{k}-\widehat{V}_{k}$

At which $\hat{V}_{t}$ is the voltage's estimated value at the $\mathrm{t}^{\text {th }}$ moment. Afterward, 
$W_{k}=W_{k-1} \widehat{V}_{k-1}$

where the weight $\mathrm{W}_{t}=\mathrm{e}^{\mathrm{j} \widehat{\omega}_{\mathrm{t}-1} \Delta \mathrm{T}}, \widehat{\omega}$ can be the angular frequency's estimated value. The model's importance is that the input consists of only one the weight vector and component vector. This complex LMS approach as formulated in [11] is employed to gauge that the condition. The system lessens the error square by recursively altering $W_{t}$ at each time period as,

$W_{k}=W_{k-1}+\mu_{k} e_{k} \widehat{V}_{k}^{*}$

where ${ }^{*}$ represents the voltage complex and $\mu$ is the variable managing the convergence and also controlling the stability.

The step size $\mu_{t}$ is diverse as in [11] for convergence of the LMS approach, when the noise presents. For complicated conditions, the equations is upgraded as,

$\mu_{k+1}=\lambda \mu_{k}+\gamma p_{k} p_{k}^{*}$

At which $p_{t}$ reflects the autocorrelation of $e_{t}$ and $e_{t-1}$ is currently calculated as

$P_{k}=\rho p_{k-1}+(1-\rho) e_{k} e_{k-1}$

At which $\rho$ is an exponential weighting factor and $0<$ $\rho<1,0<\lambda<1$ and $\gamma>0$ controlling the speed of convergence. $\mu_{t+1}$ is set to $\mu_{\max }$ or $\mu_{\min }$ If it moves over or under the upper and lower constraints respectively. All these principles have been chosen dependent on indicate numbers clarified in [11].

The motor voltage magnitude $A_{t}$ is computed at any period t from the evaluated esteem of voltage $\widehat{V}_{t}$ as,

$A_{t}=\left|\hat{V}_{t}\right|$

The slope $S_{t}$ is figured as follows,

$S_{t}=\frac{\left(A_{t}-A_{t-1}\right)}{\nabla \mathrm{T}}$

where $A_{t}$ and $A_{t-1}$ are the motor voltage magnitudes at the time interval $t$ and $t+1$ correspondingly.

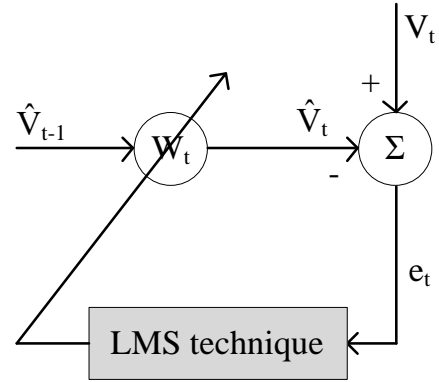

Fig. 2. Feature extraction using LMS

\section{FEED FORWARD NEURAL NETWORKS}

Feed forward neural networks (FFNNs) certainly really are an exceptional type of ANN that can be controlled. FFNNs contain a listing of processing factors termed "neurons". Neurons are dispersed across a few layers. The very first level is known as the input, also the input factors are mapped by this layer. The last level is known as the output. All levels in between the input and the output are known as hidden layers [8]. Multilayer perceptron (MLP) could be very common and popular kind of all FFNN. The neurons in FFNN have been merged at a one-way and one-way mode. The bonds have been represented by weights, and which can be real numbers within the period $[-1,1]$. Fig. 3 exhibits a FFNN neural network with just a single hidden layer. Every and every node from the network's output layer is computed in two phases. First, the formulation calculates the summation of the input.

$S_{j}=\sum_{i=1}^{n} \omega_{i j} I_{i}+\beta_{j}$

where, $I_{i}$ is input feature $\mathrm{i}$, along with $\mathrm{w}_{\mathrm{ij}}$ is that the weight of connection between Ii and the hidden neuron j. Second, the activation function can be utilized to activate the output signal of nerves depending around the significance of their summation functionality. Assorted sorts of activation features may be utilized in MLP. Utilizing the sigmoid function, that can be most commonly utilized from the literature, as that the outcome signal of $\mathrm{j}$ node from the hidden layer could be computed as,

$f_{j}(x)=\frac{1}{1+e^{-S_{j}}}$

The output of the MLP is finally obtained as,

$\hat{y}_{k}=\sum_{i=1}^{m} W_{k j} f_{i}+\beta_{k}$

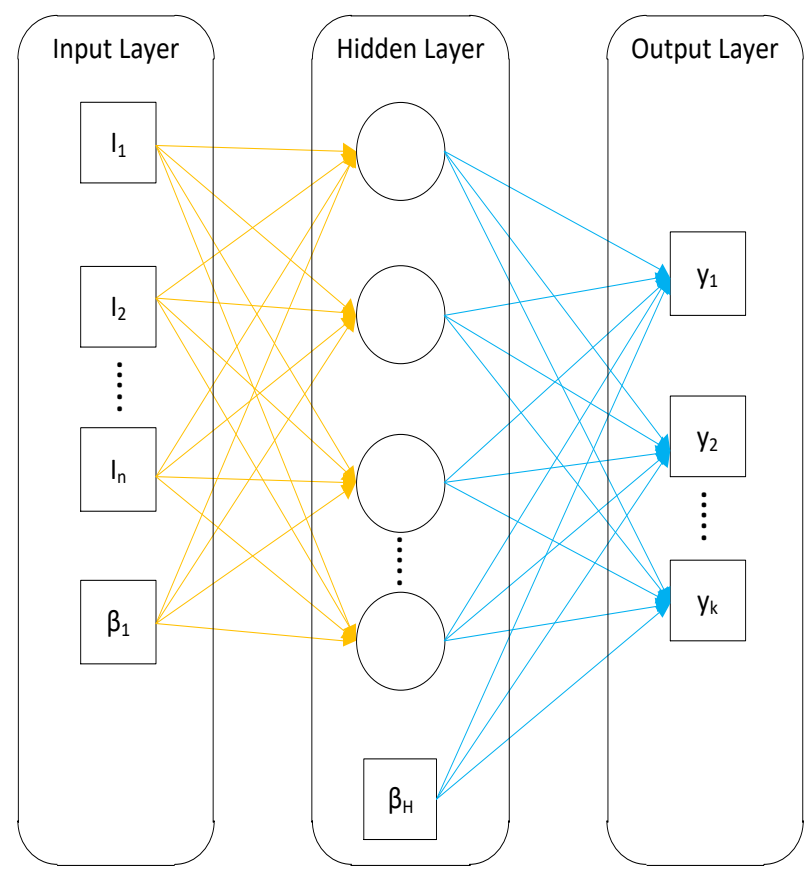

Fig. 3. Feed forward neural networks

Published By:

Retrieval Number: C5634029320/2020CBEIESP

DOI: 10.35940/ijeat.C5634.029320

Journal Website: www.ijeat.org 


\section{FFNN TRAINING USING MECA}

The mind evolution computational algorithm (MECA) described in [12] was applied. After developing the FFNN described in section 3, the application of MECA in training the FFNN has been developed in this section.

\section{A. Building an Initial Solution}

For simplicity, considered to build an initial solution consists of weight and bias. To generate the initial solution, a random constructive heuristic was used. The creation of this type of solution gives enormous potential for refinement heuristics, in particular the MECA to improve the solution to the FFNN training problem, as there is no specific criterion for selection, leaving many possibilities open. Table 1 shows the parameters used in the proposed hybrid FFNN.

\section{B. FFNN Training}

The MECA agent used to train the FFNN is depicted in Fig. 4. This process of disturbance and local search is repeated in the expectation that the entire space of solutions has been explored, bringing us the global optimum of the exploration sequencing system for the optimal weight and bias.
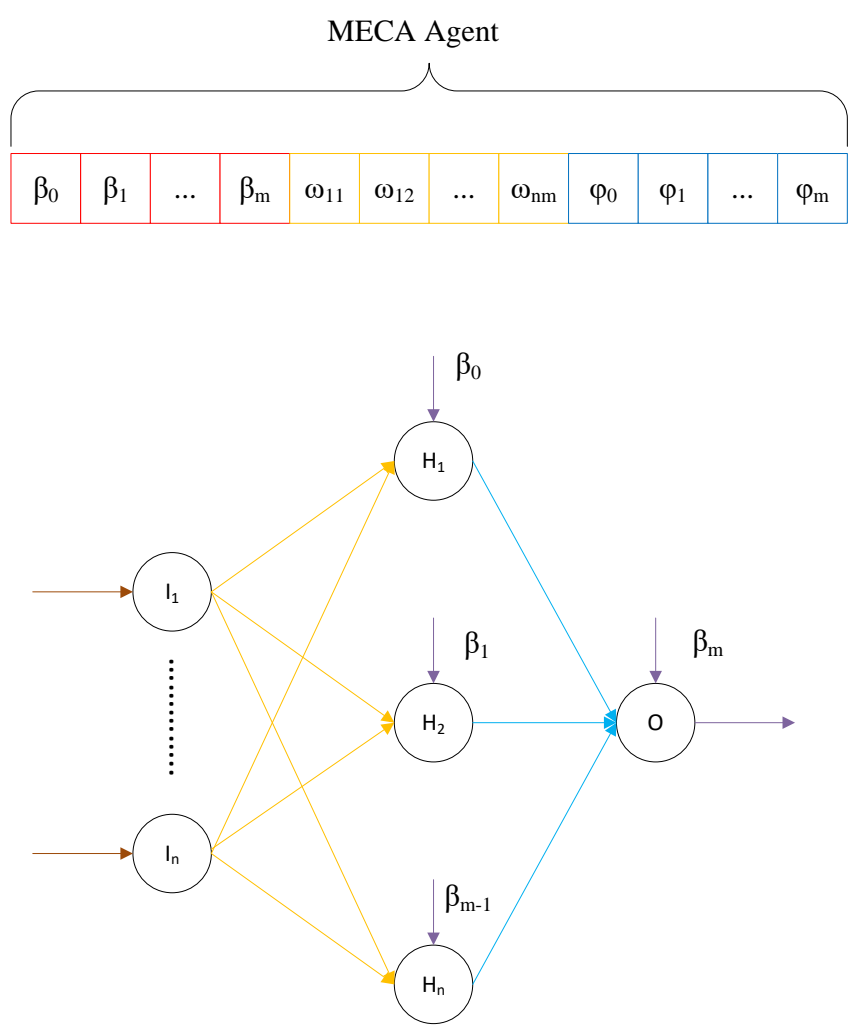

Fig. 4. MECA agent during FFNN training

In MECA-FFNN, each investigation agent has been analyzed to be a symbol of an applicant neural system. Vectors comprise a few components: a pair of weights connecting the hidden layer into the output, a group of weights linking the input to the hidden level, as well as a group of bias. The length of every vector is corresponding to this whole quantity of weights and also bias from the system, it could be computed with (19) in which $n$ is the range of inputs and $\mathrm{m}$ is that the neurons at the hidden level.

$$
\text { Individual length }=(\mathrm{n} \times \mathrm{m})+(2 \times \mathrm{m})+1
$$

To assess the objective of this established MECA representatives, we now make use of mean square error (MSE) as physical fitness functionality that's dependant on calculating the error between the true and predicted values from the established agents for working samples. MSE has been revealed in (20) where y is that the true value, ${ }^{\prime} y$ is the predicted value, and $\mathrm{n}$ is number of instances in the data set. The formula for MSE is given as,

$$
M S E=\frac{1}{n} \sum_{i=1}^{n}(y-\hat{y})^{2}
$$

Table 1. FFNN parameters

\begin{tabular}{lc}
\hline \multicolumn{2}{c}{ Structure } \\
\hline Total layers & 3 \\
Neuron on each layer & Input: 2, hidden: 5, output: 3 \\
The initial agent & Random \\
Activation functions & Tangent function \\
\hline \multicolumn{2}{c}{ Training parameters } \\
\hline Training technique & MECA \\
MSE & 1E-05 \\
\hline
\end{tabular}

\section{SIMULATION AND ANALYSIS}

Fault diagnosis of multiphase induction motor could be the pattern classification. The input design space can be sorted by the FFNN and also be implemented in classification and design recognition. It is a non linear dilemma to identify fault in multiphase induction motor with voltage amplitude and its slope. The proposed FFNN systems have been utilized to identify fault in multiphase induction motor.

\section{A. Data Generation}

Generating data employing equations evaluations has a few edges. It is potential to alter their testing and training signal's parameters in a selection and at a method that is restricted. The signals proved quite near the real circumstance. On the other hand, the different signals owned by exactly precise same categories are created and it feasible to appraise the classifiers' capacities according to FFNN [8]. The input in the failure classification system based on FFNN has been got on the basis of the model in [8]. Three types of various faults were considered. 200 examples of every class using parameters had been made for training, and also the other 200 situations have been produced for analyzing. The test and training voltage signals are sampled at 256 values / cycle, and also the frequency is $50 \mathrm{~Hz}$. Therefore, 16 power frequency cycles that make work with an interference use of an overall total of 4096 details.

Published By: 


\section{B. Simulation Results}

Data collections for both testing and training have been constructed. The dimensions right the following clarify various attributes got as a consequence of employing the LMS filter, in other words, that the total quantity of the evaluation data collection will be $2 \times 600$, at which two is size of the object in each case, and 600 is from 200 cases for the categories is multiply by 3 classes. This can be classified using MLP-NN. Considering that the classification efficacy with this system, this input features can be applied as input signal to the FFNN structure. The classification outcomes are clarified in relation to $3 \times 3$ confusion matrix as shown in Table 2 . Faults are correctly classified in diagonal elements. Off-diagonal represent wrongly classified.

The proposed FFNN has classified 588 out-of 600 failure signals as exhibited in Table 2. It follows that the success rate is all about $98 \%$ and $96.17 \%$ with and without MECA as shown in Table 3, when there is not any noise inserted to voltage waveforms.

Table 2. Results of the proposed fault diagnosis method

\begin{tabular}{lcccc}
\hline $\begin{array}{l}\text { True } \\
\text { class }\end{array}$ & $\begin{array}{l}\text { Rotor } \\
\text { fault }\end{array}$ & $\begin{array}{l}\text { Stator } \\
\text { fault }\end{array}$ & $\begin{array}{l}\text { Bearing } \\
\text { fault }\end{array}$ & $\begin{array}{l}\text { Accuracy } \\
\text { (\%) }\end{array}$ \\
\hline $\begin{array}{l}\text { Rotor } \\
\text { fault }\end{array}$ & 200 & 0 & 0 & 100 \\
$\begin{array}{l}\text { Stator } \\
\text { fault } \\
\text { Bearing } \\
\text { fault }\end{array}$ & 11 & 189 & 0 & 94.5 \\
\hline
\end{tabular}

Table 3. Comparison results

\begin{tabular}{|l|c|c|}
\hline Fault type & $\begin{array}{c}\text { FFNN with } \\
\text { MECA }\end{array}$ & $\begin{array}{c}\text { FFNN without } \\
\text { MECA }\end{array}$ \\
\hline Rotor fault & 100 & 100 \\
\hline Stator fault & 94.5 & 90 \\
\hline $\begin{array}{l}\text { Bearing } \\
\text { fault }\end{array}$ & 99.5 & 98.5 \\
\hline Overall & $\mathbf{9 8}$ & $\mathbf{9 6 . 1 7}$ \\
\hline
\end{tabular}

\section{CONCLUSION}

This article suggests a way of extracting features that are successful based on the least squares approach for the classification of faults in multiphase induction machines. The proposed classification method is done with a multilayer perceptron neural system together with the mind evolution computational algorithm. It functions as a classification algorithm based on the multilayer perceptron as well as element extraction composed of LMS filter. A comparison of performance in terms of fault diagnosis accuracy is offered to research studies. The average correct classification with and without mind evolution computation algorithm is about $98 \%$ and $96.17 \%$ for various fault signals respectively. The outcome got from the simulation analysis has been shown the potency of the proposed hybrid neural network for fault diagnosis in multi phase induction motor. Quite a many applications, for example fault classification with hybrid neural network demands a system that are great capabilities to deal in classification accuracy. This particular skill in hybrid neural neutral cannot be quickly achieved by conventional classifiers; however they are exceedingly costly and cannot be practically available.

\section{REFERENCES}

1. Tang G, Kong W, Zhang T. The Investigation of Multiphase Motor Fault Control Strategies for Electric Vehicle Application. Journal of Electrical Engineering \& Technology. 2020 Jan 1;15(1):163-77.

2. Albsmeier ED, Bornemann KR, Chiu HC, Eaton Z, Hackbarth AJ, Mauk RA, Neis JJ, Brey ED, inventors; Kohler Co, assignee. Diagnostic method for an engine-generator set. United States patent US 10,509,075. 2019 Dec 17.

3. Luo G, Hurwitz JE, Habetler TG. A Survey of Multi-Sensor Systems for Online Fault Detection of Electric Machines. In2019 IEEE 12th International Symposium on Diagnostics for Electrical Machines, Power Electronics and Drives (SDEMPED) 2019 Aug 27 (pp. 338-343). IEEE.

4. Barrero F, Bermudez M, Duran MJ, Salas P, Gonzalez-Prieto I. Assessment of a Universal Reconfiguration-less Control Approach in Open-Phase Fault Operation for Multiphase Drives. Energies. 2019 Jan;12(24):4698.

5. Ayala M, Doval-Gandoy J, Rodas J, Gonzalez O, Gregor R. Current control designed with model based predictive control for six-phase motor drives. ISA transactions. 2019 Sep 6.

6. Candelo-Zuluaga C, Riba JR, López-Torres C, Garcia A. Detection of Inter-Turn Faults in Multi-Phase Ferrite-PM Assisted Synchronous Reluctance Machines. Energies. 2019 Jan;12(14):2733.

7. Santhosh K, Neela R. optimal placement of distribution generation in micro-grid using eagle strategy with particle swarm optimizer. International Journal of Pure and Applied Mathematics. 2018;118(18):3819-25.

8. Dutta S, Chen X, Jha S, Sankaranarayanan S, Tiwari A. Sherlock-A tool for verification of neural network feedback systems: demo abstract. InProceedings of the 22nd ACM International Conference on Hybrid Systems: Computation and Control 2019 Apr 16 (pp. 262-263).

9. Annamalai B, Swaminathan ST. Diagnostics of faults in induction motor via wavelet packet transform. IOSR Journal of VLSI and Signal Processing (IOSR-JVSP). 2016:01-6.

10. Annamalai, Balamurugan and Sivakumaran Thangavel Swaminathan. "Diagnostics of Faults In Multi-Phase Induction Motor Using Wavelet Transforms." (2014).

11. Ramos, C. J., Martins, A. P., \& da Silva Carvalho, A. (2017). Power system frequency estimation using a least mean squares differentiator. International Journal of Electrical Power \& Energy Systems, 87, 166-175.

12. Chengyi S, Yan S, Keming X. Mind-evolution-based machine learning an efficient approach of evolution computation. InProceedings of the 3rd World Congress on Intelligent Control and Automation (Cat. No. 00EX393) 2000 Jun 26 (Vol. 1, pp. 118-121). IEEE.

\section{AUTHORS PROFILE}

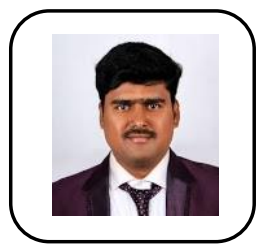

A. Balamurugan received B.E degree in Electrical and Electronics Engineering from Mookambigai College of Engineering, Trichy and M.E degree in Power electronics and drives from Periyar Maniammai University, Thanjavur in 2007 and 2012 respectively. He is a research scholar of Sathyabama institute of Science and Technology, Chennai. Currently, he is an Assistant Professor at Ariyalur Engineering College, Ariyalur. His research area is Power Electronics and drives. He is the life member of Indian Society for Technical

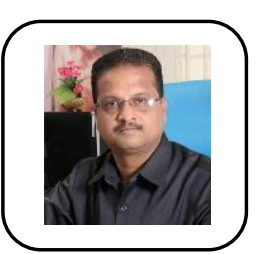
Education.

Dr. T. S. Sivakuaran received B.E degree in EEE from Annamalai University, Chidambaram, Tamilnadu in 1998 and M.Tech Degree in EEE from VIT University, Vellore, Tamilnadu in 2002. He received the Ph.D. degree in Power Electronics from Annamalai University, Chidambaram, Tamilnadu in 2009. Currently, he is a principal in Sasurie college of Engineering, Tiruppur, Department of Electrical and Electronics Engineering.

Published By: 